\title{
TIME RESPONSE OF A PARABOLIC REFLECTOR ANTENNA TO A GENERALIZED GAUSSIAN PULSE
}

\author{
R. de Oliveira ${ }^{1}$ and M. Hélier ${ }^{2}$
}

1: DRE / Université de Versailles-St-Quentin, France, rodrigo.deoliveira@lss.supelec.fr

2: LISIF / Université Pierre et Marie Curie, Paris, France, marc.helier@lis.jussieu.fr

\section{Introduction}

For a few years, interest is growing in the study of the temporal behaviour of antennas operating in pulsed mode due to the development of ultra wide band systems [1-2] or high power microwave weapons. In these applications, the parabolic reflector antenna remains an interesting case of study. Usually, methods for evaluating the electromagnetic field radiated by classical antennas are performed in the frequency domain and numerous computer codes are available for calculating the radiated electromagnetic field. Then, the time domain behaviour is investigated by means of a Fourier transform. But for the particular case of the parabolic reflector, it is more convenient to operate directly in the time domain.

In this paper, we apply the Skulkin and Turchin approach [3] to obtain a complete electromagnetic formulation of the impulse response of the parabolic reflector antenna. From it, it is easy to compute the response of the antenna to any input signal by means of a convolution product [4]. Based on the Physical Optics (PO) approximation and neglecting the wedge effects, this method leads to a fast numerical procedure for predicting the near and far fields both in time and frequency domain. To show the efficiency of the method, we have determined the radial and angular distribution of the energy density produced by a generalized gaussian pulse input signal.

\section{Geometry and assumptions}

An antenna which is usually directive in the frequency domain may behave differently in the time domain due to transients [5]. The electromagnetic field may be momentarily radiated in undesirable directions producing fratricidal effects in the case of high power microwave applications. Such a behaviour is easier to predict in the time domain when the impulse response of the antenna is known. To calculate such a response, some assumptions have to be made. The parabolic reflector is a perfectly conducting dish of radius $a$ and focal distance $b$ as shown in figure 1. In terms of cartesian coordinates $(x, y, z)$ whose origin $O$ is on the vertex, the reflector is defined by the equation:

$$
z=\frac{x^{2}+y^{2}}{4 b} \quad \text { with } \quad x^{2}+y^{2} \leq a
$$

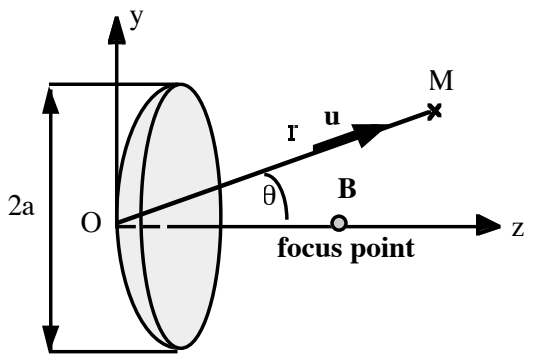

Fig. 1: Reflector geometry

The primary source is supposed to be located at the reflector focus $B(0,0, b)$ or close to it, and is a point source. Due to that assumption, the reflector itself is assumed to be localized in the far field of the point source. To ensure that condition, the input signal applied to the point source should not have any DC component and the lowest frequency $f_{0}$ of its spectrum should be such as:

$$
f_{0}>\frac{c}{b}
$$

where $c$ is the light speed in free space.

The wedge scattering is supposed to be negligible as well as the radiation due to the hidden face of the reflector. With such hypothesis, the physical optics approximation may be applied. Thus, the current density on the reflector surface depends only on the incident magnetic field $\boldsymbol{H}_{\boldsymbol{i}}$ due to the point source:

$$
\boldsymbol{J}=2 \boldsymbol{n} \times \boldsymbol{H}_{\boldsymbol{i}}
$$

where $\boldsymbol{n}$ is the normal vector to the reflector at point $P$.

\section{Theoretical approach}

The primary source is fed by an input signal $s(t)$ and the radiated field $(\boldsymbol{E}, \boldsymbol{H})$ at point $M$, defined in figure 2, is given by the convolution product:

$$
\left(\begin{array}{l}
\boldsymbol{E} \\
\boldsymbol{H}
\end{array}\right)=\left(\begin{array}{l}
\boldsymbol{E}_{\boldsymbol{i} m p} \\
\boldsymbol{H}_{\boldsymbol{i} m p}
\end{array}\right) * S(t)
$$


where $\boldsymbol{E}_{\boldsymbol{i m p}}$ is the electric field impulse response and $\boldsymbol{H}_{\boldsymbol{i} m p}$ the magnetic field impulse response at point $M$.

When $s(t)=\delta(t)$, the Dirac delta function, the radiated field is the impulse response of the antenna. The incident field on the reflector due to the primary source is given by the expression:

$$
\left(\begin{array}{l}
\boldsymbol{E}_{\boldsymbol{i}} \\
\boldsymbol{H}_{\boldsymbol{i}}
\end{array}\right)=\frac{1}{r_{1}} \delta\left(P, t-\frac{r_{1}}{c}\right)\left(\begin{array}{l}
\boldsymbol{e} \\
\boldsymbol{h}
\end{array}\right) \text { with }|\boldsymbol{e}|=\eta_{0}|\boldsymbol{h}|,
$$

$\eta_{0}=120 \pi \Omega$ is the free space impedance.

The incident electric field is normalized so that $\int_{\text {half space }} \int \frac{\left|\boldsymbol{E}_{\boldsymbol{i}}\right|^{2}}{2 \eta_{0}} d s=1$ which implies that $|\boldsymbol{h}|=\frac{1}{\sqrt{\pi \eta_{0}}}$.

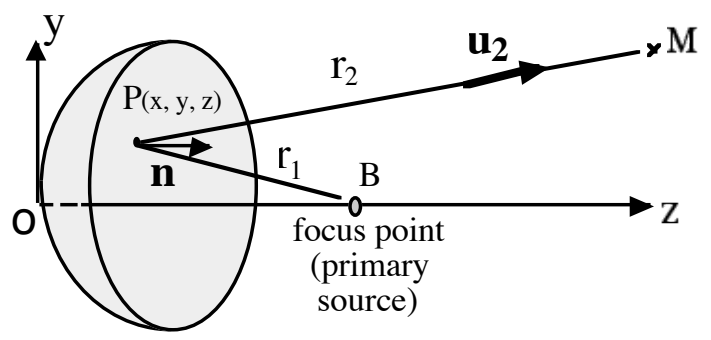

Fig. 2: Points, distances and vectors

The magnetic field integral equation (MFIE) formulation is then applied in time domain to obtain the radiated magnetic field:

$$
\begin{aligned}
& \boldsymbol{H}(M, t)=\frac{1}{4 \pi} \int_{S} \int\left[\frac{1}{r_{2}^{2}} \boldsymbol{J}\left(P, t-\frac{r_{2}}{c}\right)+\right. \\
& \left.\frac{1}{c r_{2}} \frac{\partial}{\partial t} \boldsymbol{J}\left(P, t-\frac{r_{2}}{c}\right)\right] \times \boldsymbol{u}_{2} d s
\end{aligned}
$$

Here, $S$ is the reflector surface, $\boldsymbol{r}_{2}=\boldsymbol{P} \boldsymbol{M}$ and $\boldsymbol{u}_{2}=\boldsymbol{r}_{\mathbf{2}} / r_{2}$ is a unit vector.

When replacing $\boldsymbol{J}$ by $2 \boldsymbol{n} \times \boldsymbol{H}_{\boldsymbol{i}}$ and $\boldsymbol{H}_{\boldsymbol{i}}$ by $\frac{1}{r_{1}} \delta\left(P, t-\frac{r_{1}}{c}\right) \boldsymbol{h}$, the magnetic field impulse response is obtained and may be expressed as the sum of two terms:

$$
\boldsymbol{H}_{i m p}(M, t)=\boldsymbol{H}^{\mathbf{1}}{ }_{i m p}(M, t)+\boldsymbol{H}_{i m p}^{\mathbf{2}}(M, t)
$$

with the following components:

$$
\begin{gathered}
\boldsymbol{H}^{\mathbf{1}}{ }_{i m p}=\frac{1}{2 \pi c} \iint_{S} \frac{1}{r_{1} r_{2}} \frac{\partial}{\partial t} \delta\left(P, t-\frac{r_{1}}{c}-\frac{r_{2}}{c}\right)(\boldsymbol{n} \times \boldsymbol{h}) \times \boldsymbol{u}_{2} d s \\
\boldsymbol{H}^{\mathbf{2}}{ }_{i m p}=\frac{1}{2 \pi} \iint_{S} \frac{1}{r_{1} r_{2}^{2}} \delta\left(P, t-\frac{r_{1}}{c}-\frac{r_{2}}{c}\right)(\boldsymbol{n} \times \boldsymbol{h}) \times \boldsymbol{u}_{2} d s
\end{gathered}
$$

The main component appears to be $\boldsymbol{H}^{\mathbf{1}}{ }_{i m p}$ as $\boldsymbol{H}^{\mathbf{2}}{ }_{i m p}$ decreases more rapidly with distance $r_{2}$ than $\boldsymbol{H}^{1}{ }^{1}$ imp but will not be neglected in the computation.

In order to calculate $\boldsymbol{H}^{1}{ }^{1}$ mp , it is easier to get the step response $\boldsymbol{H}_{\text {step }}^{\mathbf{1}}$ knowing that $\boldsymbol{H}_{\text {imp }}^{\mathbf{1}}=\frac{\partial}{\partial t} \boldsymbol{H}_{\text {step }}$. Then:

$$
\boldsymbol{H}_{\text {step }}^{\mathbf{1}}=\frac{1}{2 \pi c} \iint_{S} \frac{1}{r_{1} r_{2}} \delta\left(P, t-\frac{r_{1}}{c}-\frac{r_{2}}{c}\right)(\boldsymbol{n} \times \boldsymbol{h}) \times \boldsymbol{u}_{\mathbf{2}} d s
$$

Due to the Dirac delta function, the key point is that the surface integral may be expressed as a contour integral on a path $C$ implicitly defined by the equation:

$$
c t-r_{1}(x, y)-r_{2}(x, y)=0
$$

Thus, by calculating the differential $d s$ on the reflector surface:

$$
d s=p(x, y) d x d y \quad \text { with } \quad p(x, y)=\sqrt{1+\frac{x^{2}+y^{2}}{4 b^{2}}}
$$

it can be shown [6] that:

$$
\boldsymbol{H}_{\text {step }}^{\mathbf{1}}=\frac{1}{2 \pi} \int_{C} \frac{1}{r_{1} r_{2} \operatorname{grad}\left(c t-r_{1}-r_{2}\right)}(\boldsymbol{n} \times \boldsymbol{h}) \times \boldsymbol{u}_{2} p(x, y) d l
$$

Then, $\boldsymbol{H}_{\text {imp }}^{\mathbf{1}}=\frac{\partial}{\partial t} \boldsymbol{H}_{\text {step }}^{\mathbf{1}}$ and a similar approach allows to obtain:

$\boldsymbol{H}^{2}{ }_{i m p}=\frac{c}{2 \pi} \int_{C} \frac{1}{r_{1} r_{2}^{2} \operatorname{grad}\left(c t-r_{1}-r_{2}\right)}(\boldsymbol{n} \times \boldsymbol{h}) \times \boldsymbol{u}_{2} p(x, y) d l$

Last, recalling Maxwell-Faraday equation:

$$
\operatorname{curl} H=\epsilon_{0} \frac{\partial \boldsymbol{E}}{\partial t}
$$

the electric field impulse response is expressed as:

$$
\boldsymbol{E}_{\text {imp }}(M, t)=\frac{1}{\boldsymbol{\epsilon}_{0}} \mathbf{c u r l}_{\text {step }}
$$


Applying a similar approach, $\boldsymbol{E}_{\text {imp }}$ can be easily obtained as the sum of contour integrals which are given with more details in reference [6].

\section{The generalized gaussian pulse}

The primary source input signal is a generalized gaussian pulse (GGP) which is significant of ultra wide band signals [2]. It is given by the expression:

$$
\begin{aligned}
& g(t)=\frac{A}{1-\alpha}\left\{\exp \left(-\beta\left[t-\frac{\tau}{2}\right]^{2}\right)\right. \\
& \left.-\alpha \exp \left(-\alpha^{2} \beta\left[t-\frac{\tau}{2}\right]^{2}\right)\right\}
\end{aligned}
$$

$\tau$ is the pulse duration, $\alpha$ is a shape factor, $\beta$ adjusts the residual pulse level at $t=0$ or $t=\tau$, and $A$ is the pulse amplitude.

Such an impulse has theoretically a null DC component and complies with the assumption made in section II.

With such a definition, the generalized gaussian pulse is centred at $t=\frac{\tau}{2}$ where it reaches its peak value.

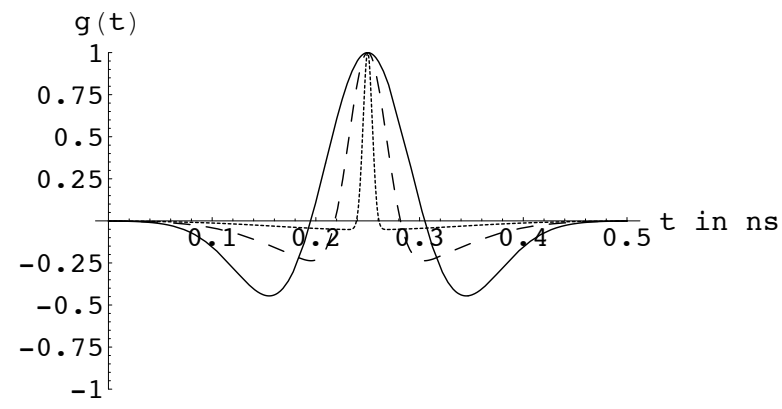

Fig. 3: Examples of GGP with different values of $\alpha$

In figure 3, some examples of generalized gaussian pulse with a unit amplitude $A$ and a 0.5 ns duration are shown for different parameter values:

$\alpha=0,05$ (dotted curve), $\alpha=0,25$ (dashed curve) and $\alpha=0,95$ (plain curve).

$\beta$ has been determined so that the residual level of the pulse is $60 \mathrm{~dB}$ below its peak value at the pulse start and stop times.

The smaller is $\alpha$, the narrower is the pulse and the wider is its bandwidth.

\section{Results}

In order to illustrate the approach, we have chosen to study a small parabolic reflector with a $20 \mathrm{~cm}$ diameter and a $10 \mathrm{~cm}$ focal distance.

First, figure 4 shows the main component of the electric field impulse response at $r=1 \mathrm{~m}$ along the reflector axis. The incident field radiated by the primary source at the reflector focus is linearly polarized with the electric field parallel to the $\mathrm{x}$-axis.

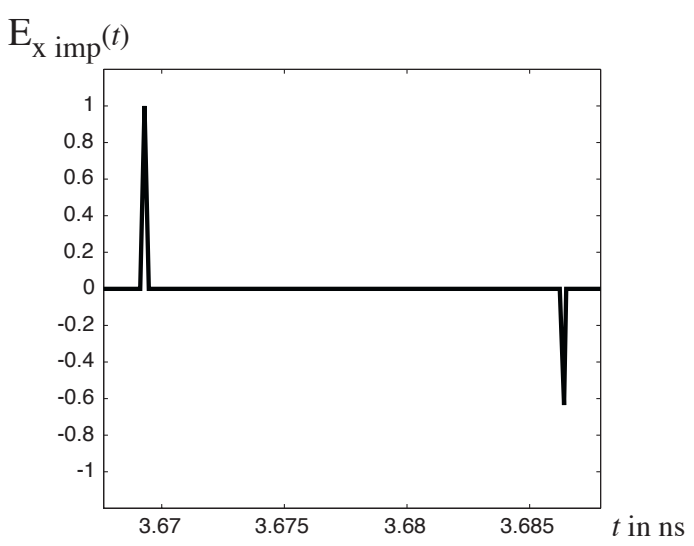

Fig. 4: Electric field impulse response (x-component)

The corresponding electric response (x-component) at the same location to a GGP with $\alpha=0,95$ and $\tau=0,5 \mathrm{~ns}$ is depicted in figure 5 . It appears to be close to the derivative of the generalized gaussian input signal as the impulse response acts as a derivative operator. The associated magnetic response (y-component) has a similar shape.

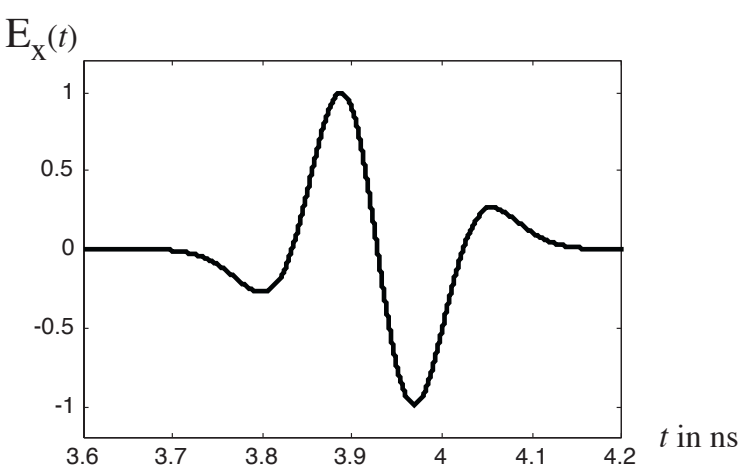

Fig. 5: Electric field response to a GGP

The corresponding surface power density response (deduced from the time dependent Poynting vector) at the same location and to the same GGP is plotted in figure 6 . 


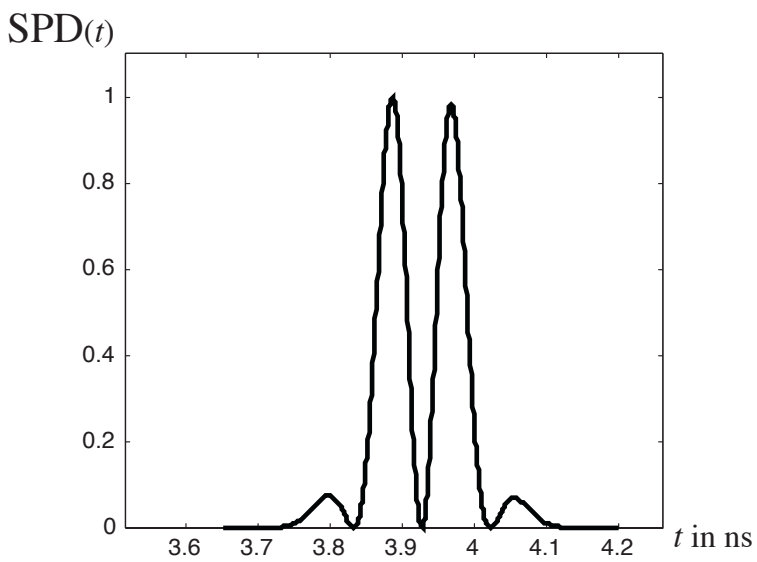

Fig. 6: Surface power density at $r=1 \mathrm{~m}$

The surface energy density at point $M(r, \theta, \varphi)$ is given by:

$$
w(M)=\int_{-\infty}^{\infty}\{\boldsymbol{E}(M, t) \times \boldsymbol{H}(M, t)\} \cdot \boldsymbol{u} d t
$$

where vector $\boldsymbol{u}$ is defined as shown in figure 1 .

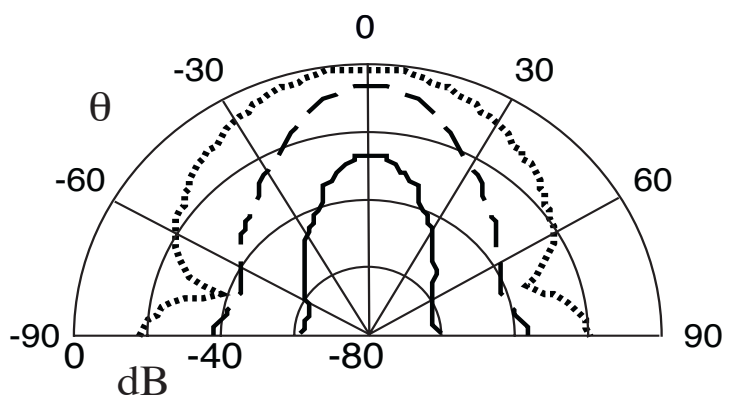

Fig. 7: Surface energy density versus $\theta$ with $\varphi=0$

Diagram of surface energy density are plotted in figure 7 at different distances from the origin: $r=14 \mathrm{~cm}$ (dotted curve), $r=54 \mathrm{~cm}$ (dashed curve) and $r=7 \mathrm{~m}$ (plain curve). The energy density decreases as the squared of the distance, as expected, and its diagram becomes more directive as distance increases.

The time evolution of the power density diagram has been computed by means of a similar approach [4] and [6].

\section{Conclusion}

Time domain computation combined with the Skulkin and Turchin approach appears to be efficient and informative to study the behaviour of a reflector antenna fed with pulses. The knowledge of the impulse response allows to calculate the response of the antenna to any input signal like a generalized gaussian pulse meaningful of ultra wide band signals. As the classical radiation pattern or gain concepts are not relevant in the time domain, the surface energy density diagram may be helpful to understand the way the antenna operates. Such a method could be extended to other kinds of antennas like multi-reflector antennas.

\section{Acknowledgments}

The authors are very grateful to Pr. Walid Tabbara, Dr. Françoise Brouaye and Pr. Richard Dusséaux for fruitful discussions.

\section{References}

[1] A. Sibille, C. Roblin, S. Bories, A.C. Lepage, X. Begaud, "Conception et caractérisation d'antennes ULB pour communications multimédia haut débit », REE, n4, avril 2004, pages 73 à 80, ISSN 1265-6534.

[2] M. Granger, « Radar ULB : impulsionnel ou bande synthétique ? », REE, n4, avril 2004, pages 64 à 69, ISSN 1265-6534.

[3] Sergey P. Skulkin, "Transient Fields of Rectangular Aperture Antennas in Ultra-Wideband Short-Pulse Electromagnetics”, Volume 3, pp. 57-63, Edited by Carl E. Baum, Lawrence Carin, Alexander P. Stone, Plenum, 1997, ISBN 0-306-45593-5.

[4] R. de Oliveira, M. Hélier, "Time domain analysis of the radiation pattern of a parabolic reflector antenna”, JINA 2004, Proceedings, pp. 68-69, November 8-10, 2004, Nice, France.

[5] R. C. Hansen, "Short-Pulse excitation of reflector antennas”, IEE Proceedings-H, Vol. 134, No. 6, pp 557-559, December 1987.

[6] R. de Oliveira, M. Hélier, “Le rayonnement d'une parabole par une approche temporelle semi-analytique”, accepted paper to be published in 2005 in the "Annals of telecommunications” journal. 